\title{
DICCIONARIO HISTÓRICO DEL ESPAÑOL MODERNO DE APARATOS DE FÍSICA EXPERIMENTAL: DOCUMENTACIÓN DE LOS TÉRMINOS DEL SIGLO XVIII ${ }^{1}$
}

\author{
Antoni Nomdedeu Rull y Sandra Iglesia Martín \\ Universitat Rovira i Virgili \\ Grupo NEOLCYT \\ antonio.nomdedeu@urv.ca; sandra.iglesia@urv.cat
}

Recibido: 25 abril 2012; Aceptado: 28 diciembre 2012

Cómo citar este artículo/Citation: Nomdedeu Rull, Antoni e Iglesia Martín, Sandra (2013), "Diccionario Histórico del Español moderno de aparatos de física experimental: documentación de los términos del siglo XVIII", Asclepio 65 (2): p020. doi: http://dx.doi.org/10.3989/ asclepio.2013.20

RESUMEN: El objetivo de este estudio es presentar la documentación de los términos referidos a aparatos en textos de Física experimental del siglo XVIII y realizar su análisis léxico y lexicográfico. A partir de la metodología de trabajo del grupo NEOLCYT (http://seneca.uab.es/neolcyt), se pretende sentar las bases para la elaboración de un Diccionario histórico del español moderno de aparatos de Física experimental (DHEMAFE) como parte del Diccionario histórico del español moderno de la ciencia y de la técnica (DHEMCYT) del grupo NEOLCYT.

Los resultados del análisis de los datos muestran que los textos analizados tuvieron una influencia decisiva en el desarrollo de la Física experimental en España y permitieron la divulgación de neologismos referidos a aparatos en este periodo. Los textos que conforman el corpus de análisis presentan un porcentaje muy elevado de términos no documentados ni en nuestra lexicografía $(31,37 \%)$ ni en el CORDE $(83,4 \%)$, datos que refuerzan la necesidad de seguir en esta línea de investigación de cara a la elaboración del DHEMAFE.

PALABRAS CLAVE: Diccionario histórico; aparatos; Física experimental; Nollet; La Fond. DICCIONARIO HISTÓRICO DEL ESPAÑOL MODERNO DE APARATOS DE FÍSICA EXPERIMENTAL: DOCU-
MENTATION OF THE TERMS RELATING TO DEVICES IN THE $18^{\text {TH }}$ CENTURY

\begin{abstract}
The aim of this study, as an example of group work methodology NEOLCYT (http://seneca.uab.es/neolcyt), is to document terms relating to devices in the $18^{\text {th }} \mathrm{c}$. Spanish texts on experimental physics and their lexicographical and lexical analysis. It attempts to state the methodological bases to create the Diccionario histórico del español moderno de aparatos de Física experimental (DHEMAFE) as part of the Diccionario histórico del español moderno de la ciencia y de la técnica (DHEMCYT) by NEOLCYT group. The results of the data analysis show that the analyzed texts are essential because they had a decisive influence on the development of Experimental Physics in Spain and allowed disclosure of neologisms in that period, relating to machines. The texts of our corpus collect a very high percentage of undocumented terms, both in our lexicography $(31,37 \%)$ and in the CORDE $(83,4 \%)$. This fact reinforces the need to continue this line of research trigger in the development of DHEMAFE as part of DHEMCYT.
\end{abstract}

KEY WORDS: Historical Dictionary; Devices; Experimental Physics; Nollet; La Fond.

Copyright: (c) 2013 CSIC. Este es un artículo de acceso abierto distribuido bajo los términos de la licencia Creative Commons Attribution-Non Commercial (by-nc) Spain 3.0. 


\section{INTRODUCCIÓN}

El objetivo de este estudio es analizar las voces referidas a aparatos en los textos de Física experimental en español del siglo XVIII. Tras la recopilación de los términos, mostramos su tratamiento lexicográfico en los diccionarios generales de español para comprobar, sobre todo, la incorporación de las voces analizadas y poder contar, de este modo, con los datos lexicográficos a nuestra disposición acerca de su significado. También realizamos una breve incursión en la lexicografía especializada de la época como contrapunto a la información en diccionarios generales, aunque el análisis en profundidad de este tipo de repertorios lexicográficos se pospone para estudios posteriores. Los resultados de esta investigación, así como los que mostraremos en otras sucesivas que persiguen el mismo objetivo, pero en textos del siglo XIX, constituirán las bases del Diccionario histórico del español moderno de aparatos de Física experimental (DHEMAFE), parte del Diccionario histórico del español moderno de la ciencia y de la técnica (DHEMCYT) elaborado por el grupo NEOLCYT.

El factor que justifica la elaboración del DHEMAFE es que en el siglo XVIII los aparatos fueron decisivos para poder llevar a cabo las experimentaciones que se explicaban en los libros. La construcción de dichos aparatos se perfeccionó progresivamente, «permitiendo los progresos que se realizaron a lo largo de la centuria en todas las ciencias: sin instrumentos no habría observaciones, ni averiguación de las hipótesis, etc. Fueron los ayudantes serviles de los sabios» (Clément, 1993, p. 58).

La metodología de trabajo seguida es la propia de las investigaciones del grupo NEOLCYT. Por un lado, documentamos las fuentes fundamentales a partir de la representatividad de las obras y de los autores. Por otro lado, contextualizamos las obras seleccionadas en la historia de la ciencia y de la técnica en general y en la historia de la Física experimental de la época. Todos estos datos se consideran necesarios para abordar con garantías el estudio diacrónico de este tipo de léxico.

\section{LA FÍSICA EXPERIMENTAL EN EL SIGLO XVIII}

El siglo XVIII presentó avances científicos que heredaron la ampliación de la experimentación producida a finales del siglo XVII. Desde que Newton (16421727) publicara Philosophiae Naturalis Principia Mathematica en 1687 -en donde formuló tres principios del movimiento y la Ley de gravitación universal, según los cuales todos los fenómenos podían verse mecánicamente-, todas las investigaciones de finales del siglo XVII y todo el siglo XVIII se basaron en sus ideas. Este siglo representó el triunfo del newtonianismo frente al cartesianismo, de modo que los métodos de la lógica formal se fueron reemplazando por los de las ciencias naturales, basados en la experimentación para descubrir las leyes naturales. Se pasó, así, de la Física escolástica o especulativa a la Física experimental.

Las corrientes más importantes de la Física experimental del siglo XVIII, en todos los casos newtonianas, se gestaron fundamentalmente en Francia y en Holanda, y se extendieron luego por diversos países de Europa. Uno de los grandes difusores de la Física experimental en Francia fue Nollet. El contenido de su obra no difería del de los autores holandeses, pero la aplicación de principios como la aceptación de las ideas cartesianas en sus explicaciones sobre los fenómenos gravitatorios, eléctricos o magnéticos, supuso un distanciamiento con respecto a los holandeses (Guijarro, 2001a, p. 114). En Holanda, la Física experimental defendida por Sanguerdius (1664-1724), Boerhave (1668-1738), 'sGravesande (1688-1742) y Petrus van Musschenbroek (1692-1761) «aspiraba a convertirse en una alternativa programática tanto del aristotelismo como del cartesianismo» (Guijarro, 2001a, p. 118). De este modo, los holandeses buscaban desarrollar un sistema de enseñanza de la Física experimental basado en las prácticas de las investigaciones experimentales y no determinado por su posición en el marco de un sistema filosófico (Guijarro, 2001a, p. 118).

La recepción en España de los textos científicos y técnicos se produjo por medio de textos traducidos que, fueran o no de autores franceses, en este período llegaban del francés ${ }^{3}$, lo que conlleva que el estudio de la lengua de la ciencia y de la técnica en español en ese momento deba afrontarse como una labor de traducción permanente ${ }^{4}$. Los hechos que provocaron el triunfo de la nueva Física en España fueron la exactitud de las teorías de Newton y la introducción de los experimentos llevados a cabo por medio de las máquinas eléctricas (Vernet, 1975, p. 174). Pero la difusión del sistema newtoniano, todavía prohibido por la Iglesia, fue lenta. Con la llegada de la dinastía borbónica al trono español se favoreció el desarrollo científico, aunque fuera con fines estratégicos y militares ${ }^{5}$, lo que «permitió fomentar (ilustración) y vigilar (despotismo) el progreso científico» (Clément, 1993, p. 29). Se crearon academias y sociedades científicas según el modelo de la Accademia del Cimento de Florencia, la Royal Society de Londres (1662) y la Académie des Sciences de París (1666), cátedras, centros de enseñanza científica y técnica y sociedades económicas. Entre tanto, la estructura de las universidades españolas permanecía intacta durante la primera mitad del siglo XVIII, debido al peso de una tradición (Sánchez Ron, 1988, p. 9) todavía asentada en la escolástica o doctrina aristotélica (Clément, 1993, p. 30), lo que conlleva en ese momento que la ciencia newtoniana se introduzca "oficialmente en la univer- 
sidad española con un considerable retraso respecto a la europea» (Clément, 1993, p. 30). Con todo, habría que esperar a los planes de estudios promovidos por los ministros de Carlos III (1716-1788) para presenciar la renovación oficial de los estudios y la superación del viejo aristotelismo, concretamente con una reforma aprobada por el Consejo de Castilla que permitió que, a partir de 1770 , la nueva ciencia pudiese ser introducida en las aulas, aunque como Física de aparatos, dejando los aspectos teóricos, la Física filosófica, en manos de las corporaciones religiosas ${ }^{6}$.

Las instituciones españolas que impulsaron con más firmeza la presencia de la Física experimental en los planes de estudio universitarios fueron el Colegio Imperial (1624), el Seminario de Nobles de Madrid (1725), la Conferencia Físico-matemática experimental de Barcelona (1764) y los Reales Estudios de Madrid $(1770)^{7}$. De estos centros, que contaron con enseñanzas de Física experimental, el más destacado fue los Reales Estudios de Madrid, que aprobó un nuevo plan de estudios con cátedras de Matemáticas, Lógica y Física experimental, entre otras disciplinas (Guijarro, 2001a, pp. 133-134). Pero también tuvieron una gran repercusión, por ejemplo, el Seminario de Nobles de Madrid, que contó con las enseñanzas del profesor Antonio Zacagnini, traductor de las Lecciones de physica experimental (1757) de Nollet, y del profesor de Arte Militar y Delineación Tadeo Lope Aguilar, traductor del Elementos de Física Teórica y Experimental de Sigaud de La Fond, o la Conferencia Físico-matemática experimental de Barcelona, que se dedicó al estudio de la obra de Musschenbroek.

La nueva Física se estudiaba con máquinas, que se importaban de países como Holanda, Francia e Inglaterra, y propició la aparición de una nueva artesanía, la de constructor de aparatos científicos destinados a nutrir los laboratorios (Vernet, 1975, p. 174). Casi todos los aparatos que se importaban y, posteriormente, se fabricaban en España estaban inspirados en el Essai de Physique de Musschenbroek (Vernet, 1975, p. 175). Por ejemplo, desde los primeros textos de Física experimental en español, como en Lecciones de physica experimental de Nollet $(1757)^{8}$, se evidencia que la finalidad primordial de dicha materia fue ofrecer un modelo mecánico que diera cuenta de los hechos dentro del marco conceptual corpuscularista, factor que justifica la necesaria atención a los términos que denominan aparatos en estos textos ${ }^{9}$.

\section{LOS TEXTOS SELECCIONADOS}

Los textos seleccionados, dos traducidos del francés y cuatro producciones originales en español, fueron decisivos para el establecimiento paulatino de la nueva Física, pues presentaban numerosas novedades, se emplearon en diversas instituciones y los cargos que desempeñaron sus autores fueron de especial influencia ${ }^{10}$.

- Herrero y Rubira, Antonio María (1738), Physica moderna, experimental, sistematica: donde se contiene lo mas curioso y util de quanto se ha descubierto en la naturaleza, Madrid.

- Piquer, Andrés (1745), Física moderna, racional y experimental, Valencia, Oficina de Pasqual García. (Este manual tuvo tres ediciones: 1745, 1780 y 1790$)$.

- Jorge Juan y Antonio de Ulloa (1748), Observaciones astronómicas, y physicas, Madrid, Juan de Zúñiga.

- Jean-Antoine Nollet (1757), Lecciones de physica experimental (traducción de Antonio Nicolás Zacagnini Colón).

- Carlos Francisco Amellér (1788), Elementos de geometría, y física experimental, para el uso, $e$ instruccion de los alumnos del Real Colegio de Cirugía de Cádiz, Cádiz, Imprenta de D. Manuel Ximenez Carreño.

- Joseph-Aignan Sigaud de La Fond (1787-891, $\left.1799^{2}\right)$, Elementos de Física Teórica y Experimental (traducción de Tadeo Lope y Aguilar de los Élements de physique théorique et expérimentale, 1777).

En cuanto a los textos escritos originalmente en español, hemos analizado los de Antonio María Herrero y Rubira (1738), Andrés Piquer (1745), Jorge Juan y Antonio de Ulloa (1748) y Carlos Francisco Amellér (1788). Los dos textos traducidos son de Jean-Antoine Nollet (1757) y Sigaud de La Fond (1799), los autores más representativos de este período, el segundo discípulo del primero, cuyos trabajos se divulgaron en España y, casi de manera simultánea, en América Latina ${ }^{11}$.

La heterogeneidad de los textos de Física experimental queda demostrada en la variedad de ramas que trataban. Como se observa en la tabla 1, la Física experimental del siglo XVIII consideraba, principalmente, el estudio de la luz, el calor, el magnetismo y la electricidad, campo este que alcanzó un desarrollo mayor al resto, convirtiéndose en un ámbito de estudio modélico para los físicos experimentales.

Estos campos son los que constituirían la Física experimental, lo que permitía que se diferenciara de la Física general, ciencia cuantitativa y exacta, equivalente a la Mecánica. Aunque la Física experimental se reconoció en el siglo XVIII gracias a la reorganización de l'Académie des Sciences de París en 1785, cuando la Physique entró a formar parte de la división de ciencias matemáticas, habría que esperar hasta el siglo XIX para hallarse con una Física como una disciplina 
Tabla 1. Ramas de la Física experimental tratadas en los textos del siglo XVIII

\begin{tabular}{|c|c|c|c|c|c|}
\hline $\begin{array}{l}\text { Herrero y Rubira } \\
\text { (1738) }\end{array}$ & Piquer (1745) & Juan y Ulloa (1748) & Nollet (1757) & Amellér (1788) & La Fond (1799) \\
\hline $\begin{array}{l}\text { I. De la naturaleza, } \\
\text { existencia y } \\
\text { principios de los } \\
\text { cuerpos }\end{array}$ & $\begin{array}{l}\text { Tratado I. De la } \\
\text { Fisica, su utilidad, } \\
\text { y modo de } \\
\text { aprenderla. }\end{array}$ & $\begin{array}{l}\text { Libro I. Observaciones } \\
\text { sobre la maxima Obliquidad } \\
\text { Eliptica }\end{array}$ & $\begin{array}{l}\text { Tomo I. } \\
\text { Leccion Primera. } \\
\text { Leccion Segunda. } \\
\text { De la Porosidad, } \\
\text { Compresibilidad, y } \\
\text { Elasticidad de los cuerpos. } \\
\text { Leccion Tercera. } \\
\text { De la mobilidad de los } \\
\text { cuerpos; del movimiento, } \\
\text { de sus propiedades, y de } \\
\text { sus leyes. } \\
\text { Leccion Cuarta. Prosiguen } \\
\text { las leyes del movimiento } \\
\text { simple. }\end{array}$ & $\begin{array}{l}\text { Elementos de Geometría. } \\
\text { Exordio. } \\
\text { Libro I.. De las lineas, y de } \\
\text { los Angulos } \\
\text { Libro II.o De los triangulos } \\
\text { Libro IIIO. Del circulo } \\
\text { Libro IV.o De los solidos } \\
\text { Libro V.o De las } \\
\text { proporciones } \\
\text { Libro VI.o Practica de la } \\
\text { geometria }\end{array}$ & $\begin{array}{l}\text { Tomo I. } \\
\text { Seccion I } \\
\text { De los principios de } \\
\text { los cuerpos, de sus } \\
\text { combinaciones, y de la } \\
\text { coerencia entre las partes } \\
\text { integrantes de los mixtos } \\
\text { Sección II } \\
\text { De las propiedades } \\
\text { generales de los cuerpos } \\
\text { Sección III } \\
\text { Del movimiento }\end{array}$ \\
\hline II. De los Elementos & $\begin{array}{l}\text { Tratado II. De los } \\
\text { principios del Ente } \\
\text { natural. }\end{array}$ & $\begin{array}{l}\text { Libro II. Observaciones de } \\
\text { Latitud }\end{array}$ & $\begin{array}{l}\text { Tomo II. } \\
\text { Leccion Quinta. Sobre el } \\
\text { movimiento compuesto, y } \\
\text { sobre las Fuerzas Centrales. } \\
\text { Leccion Sexta. De la } \\
\text { gravedad de los cuerpos. } \\
\text { Leccion Septima. De la } \\
\text { Hydrostatica. } \\
\text { Leccion Octava. Prosigue la } \\
\text { misma materia. }\end{array}$ & $\begin{array}{l}\text { Lecciones de fisica } \\
\text { experimental. } \\
\text { Proemio } \\
\text { Leccion I.a } \\
\text { Seccion Primera. Que trata } \\
\text { de la fisica, y su objeto } \\
\text { Seccion 2.a De la extensión } \\
\text { y divisibilidad de los } \\
\text { cuerpos } \\
\text { Leccion II. a De la figura de } \\
\text { los cuerpos }\end{array}$ & $\begin{array}{l}\text { Tomo Il. } \\
\text { Sección primera } \\
\text { De la estática ó de las } \\
\text { máquinas } \\
\text { Sección } \mathrm{V} \text { [sic] } \\
\text { De la Hydrostática. } \\
\text { Sección VI } \\
\text { Del agua }\end{array}$ \\
\hline $\begin{array}{l}\text { III. De las qualidades } \\
\text { de los cuerpos }\end{array}$ & $\begin{array}{l}\text { Tratado III. Del } \\
\text { Movimiento. }\end{array}$ & $\begin{array}{l}\text { Libro III. Observaciones } \\
\text { de las Inmersiones, y } \\
\text { Emersiones de los Satelites } \\
\text { de Jupiter, como de los } \\
\text { Eclipses de Luna }\end{array}$ & $\begin{array}{l}\text { Tomo III. } \\
\text { Lección Novena: Sobre la } \\
\text { Mechanica. } \\
\text { Lección Decima: Sobre la } \\
\text { naturaleza, y propiedades } \\
\text { del Ayre. } \\
\text { Lección XI: Prosiguen las } \\
\text { propiedades del ayre. }\end{array}$ & & $\begin{array}{l}\text { Tomo III. } \\
\text { Sección VII } \\
\text { Del aire. }\end{array}$ \\
\hline $\begin{array}{l}\text { IV. Del movimiento, } \\
\text { y quietud }\end{array}$ & $\begin{array}{l}\text { Tratado IV. De los } \\
\text { Elementos. }\end{array}$ & $\begin{array}{l}\text { Libro IV. Sobre la Dilatacion, } \\
\text { y Compresion de los } \\
\text { Metales }\end{array}$ & $\begin{array}{l}\text { Tomo IV. } \\
\text { Lección XII: Sobre la } \\
\text { naturaleza, y propiedades } \\
\text { del agua. } \\
\text { Lección XIII: De la } \\
\text { naturaleza, y propiedades } \\
\text { del fuego. }\end{array}$ & & $\begin{array}{l}\text { Tomo IV. } \\
\text { Sección } X \text {. } \\
\text { Del fuego. }\end{array}$ \\
\hline $\begin{array}{l}\text { V. De las } \\
\text { propiedades del } \\
\text { movimiento }\end{array}$ & $\begin{array}{l}\text { Tratado V. De las } \\
\text { Piedras. }\end{array}$ & $\begin{array}{l}\text { Libro V. Sobre las } \\
\text { Experiencias del Barometro } \\
\text { simple }\end{array}$ & $\begin{array}{l}\text { Tomo V. } \\
\text { Lección XIV: Continuacion de } \\
\text { las propiedades del fuego. } \\
\text { Lección XV: Sobre la Luz. }\end{array}$ & & $\begin{array}{l}\text { Tomo V } \\
\text { Seccion XII } \\
\text { De la Meteorológia. } \\
\text { Seccion XIII. Del sistéma del } \\
\text { Mundo. } \\
\text { Sección XIV. De las causas } \\
\text { físicas de los movimientos } \\
\text { celestes }\end{array}$ \\
\hline $\begin{array}{l}\text { VI. De las leyes del } \\
\text { movimiento }\end{array}$ & & $\begin{array}{l}\text { Libro VI. De la Velocidad } \\
\text { del Sonido }\end{array}$ & $\begin{array}{l}\text { Tomo VI. } \\
\text { Lección XVI: Sobre la Luz. } \\
\text { Lección XVII: Siguen las } \\
\text { Propiedades de la Luz. }\end{array}$ & & $\begin{array}{l}\text { Tomo VI } \\
\text { Notas a la sección I. } \\
\text { Notas a la sección II. } \\
\text { Notas a la sección III. } \\
\text { Notas a la sección IV. } \\
\text { Notas a la sección V. } \\
\text { Notas a la sección VII. } \\
\text { Notas a la sección X. } \\
\text { Notas a la sección XII. } \\
\text { Notas a la sección XIII. }\end{array}$ \\
\hline $\begin{array}{l}\text { VII. De la dureza, } \\
\text { blandura, y } \\
\text { elasticidad de los } \\
\text { cuerpos }\end{array}$ & & $\begin{array}{l}\text { Libro VII. De la medida } \\
\text { del grado de Meridiano } \\
\text { contiguo al Equador }\end{array}$ & & & \\
\hline $\begin{array}{l}\text { VIII. Del Choque de } \\
\text { los cuerpos }\end{array}$ & & $\begin{array}{l}\text { Libro VIII. De las } \\
\text { Experiencias del Pendulo } \\
\text { simple, y conclusion de la } \\
\text { Figura de la Tierra }\end{array}$ & & & \\
\hline $\begin{array}{l}\text { IX. De la Pesadèz de } \\
\text { los cuerpos }\end{array}$ & & $\begin{array}{l}\text { Libro IX. De la Navegacion } \\
\text { sobre la Elipsoide }\end{array}$ & & & \\
\hline $\begin{array}{l}\text { X. Del tiempo, lugar, } \\
\text { y demás qualidades } \\
\text { de los cuerpos }\end{array}$ & & & & & \\
\hline
\end{tabular}


unificada y autónoma, separada hasta entonces de la Física general.

Entre los textos que presentaban los principios básicos de la Física newtoniana, aparecieron, en primer lugar, el de Herrero y Rubira (1738) y el de Piquer (1745), manuales que supusieron la modernización de la investigación físico-tecnológica (Maldonado y García, 2002, p. 148), si bien, desde el punto de vista lingüístico, fueron obras en donde la aparición de denominaciones referidas a aparatos era escasa, al centrarse sobre todo en explicar las observaciones de la naturaleza realizadas hasta el momento.

En cuanto a la importancia de los textos, a continuación se especifican los rasgos que justifican su consideración para este estudio.

Physica moderna, experimental, sistematica (1738) de Antonio M. Herrero y Rubira (1714-1767) -también autor de la obra en tres volúmenes Diccionario universal francés y español (1744), Madrid, Imprenta del Reyno- es la primera obra de Física moderna editada en España. El autor, como afirma en esta obra, era enemigo del aristotelismo y polemiza con Losada, Tosca y Feijoo, pero no se separa de la Teología. Por el contenido del libro, constituye un claro exponente del cartesianismo aprendido durante su estancia en Toulouse, motivo por el cual no tuvo continuidad al verse superado por los textos de orientación newtoniana que le sucederían.

El libro de Piquer (1745) introdujo la Física mecanicista bajo los principios de Descartes y de Newton. Destacó la importancia de Piquer (1711-1772) en el contexto médico de la época: fue Socio de Honor de la Real Academia Medica-Matritense y en 1751 fue nombrado Médico de la Cámara por parte de Fernando VI. Hasta este momento ocupó la cátedra de Anatomía de la Universidad de Valencia. Su obra se dirigía "con especial cuidado à los Medicos» (Piquer, Prologo [sic]) y en ella se exponían «los principales fundamentos de la Fisica experimental» (Piquer, Prologo [sic]). Es una obra circunscrita en la Física aristotélica porque, por un lado, "se enseña en todas las Universidades, y Claustros de España, y son muchos los autores españoles que la tratan con estension» (Piquer, Prologo [sic]) y, por el otro, "todo quanto en esta Obra digere, lo sujeto humildemente al juicio, y correccion de la Santa Iglesia Catolica Romana» (Piquer, Prologo [sic]). Asimismo, estamos ante una obra claramente ecléctica, en donde Piquer trató de conciliar lo antiguo y lo moderno en el marco de la tradición cristiana (Miralles y Miralles, 2007, p. 172) ${ }^{12}$ e intentó «ofrecer un tratado sistemático y didáctico de todas las materias relacionadas con esta disciplina» (Peset y Lafuente, 1981 , p. 277).

Los textos propiamente experimentales, en los que se hallan descripciones detalladas de los experimentos llevados a cabo, son los cuatro siguientes.
Leçons de physique expérimentale de Jean-Antoine Nollet (1743-48), cuya traducción al español fue llevada a cabo por Antonio Colón Zacagnini en 1757 (Lecciones de physica experimental), reflejó el cambio que se produjo en la Física en esos momentos, a raíz de las ideas de Newton, superando la concepción escolástica, y constituyó una obra fundamental para la divulgación de la Física en España y, por tanto, para la introducción y la fijación de las voces físicas en español. Se «impuso rápidamente como libro de texto en las clases de física que se crean en las Sociedades de Amigos del País o en las Academias» (Vernet, 1975, p. 174) y fue recomendado por Antonio Caballero y Góngora y por Eloy Valenzuela, dos reconocidos profesores de Física de finales del siglo XVIII, en sus programas de estudio. Su amplio uso se debió a la riqueza de experimentos que presentaba, mediante la indicación de la preparación, los efectos y sus aplicaciones, lo que respondía a la insistencia que se hacía sobre la experimentación en esa época. Tuvo nueve ediciones en francés ${ }^{13}$ y una en español ${ }^{14}$.

Nollet ha sido una figura particularmente desatendida a pesar de que lideró la Física francesa durante décadas (Sellés, 2001, p. 165) y fue uno de los principales difusores de la Física experimental en Francia (Guijarro, 2001a, p. 114). Fue miembro de la Royal Society of London ${ }^{15}$ desde 1734 , primer profesor de Física experimental de la Universidad de París (17351760) y presidente de la Académie des sciences ${ }^{16}$ de Francia desde $1762^{17}$. Abrió «en París un curso público de Física experimental, que tuvo una inmensa repercusión en la alta sociedad» (Clément, 1993, p. 16). En consecuencia, Luis XV «decidió fundar para el abate una cátedra de Física experimental en el Colegio de Navarra (1753)» (Clément, 1993, p. 16). Fue un científico innovador que descubrió la difusión de los líquidos, observó cómo el sonido podía propagarse en un medio líquido, inventó un electroscopio con láminas de oro (1748) y realizó reformas en la botella de Leyden, inventada por Musschenbroek, a la cual reemplazó el agua que contenía el recipiente por láminas de estaño o de cobre. Las concepciones de la Física experimental existentes se dividían entre el uso de la hipótesis mecanicista y el de la hipótesis sustancialista (o materialista). Y Nollet era uno de los principales representantes de la hipótesis mecanicista con sus trabajos sobre electricidad (Guijarro, 2001b, p. 198).

Por lo que respecta al traductor de la obra de Nollet al español ${ }^{18}$, Antonio Nicolás Zacagnini Colón (1723 Cádiz-1810 Génova), destaca que estudió filosofía y teología en España hasta 1740, año en que siguió los cursos de matemáticas y de Física experimental del Abate Nollet, el que fuera su catedrático en París. Enseñó la Física que había aprendido desde su posición de catedrático en el Real Seminario de Nobles de Ma- 
drid $^{19}$ a partir de 1757 y empezó a impartir conferencias públicas sobre este tema en $1758^{20}$. En 1762 se le nombró maestro de los Infantes, los hijos del rey Carlos III.

Las Observaciones astronómicas, y physicas de Jorge Juan y Antonio de Ulloa (1748) es «una obra experimental basada en principios newtonianos" (Rodríguez Ballesteros, 2004, p. 478) y en ella se describen los trabajos científicos al estilo de un informe detallado con resultados.

Jorge Juan (1713-1773) fue Capitán, desde 1752, de la Real Compañía de Guardiamarinas de Cádiz, en la Academia de Nobles Artes de San Fernando fue Académico de Honor (1767), Académico de Mérito (1768) y Consiliario (1770), y fue Director del Real Seminario de Nobles de Madrid desde 1770 (González de Posada, 2008, p. 117). Fundó hacia 1753 el primer observatorio astronómico español en el castillo de Cádiz. Antonio de Ulloa (1716-1795), por su parte, fue el fundador del Estudio y Gabinete de Historia Natural, antecesor del Real Gabinete de Historia Natural, actual Museo Nacional de Ciencias Naturales, del Observatorio Astronómico de Cádiz y el primer laboratorio de metalurgia del país, así como miembro de la Real Academia sueca, la Academia de Berlín y correspondiente de la Real Academia de Ciencias de París.

Elementos de geometría, y física experimental, para el uso, e instruccion de los alumnos del Real Colegio de Cirugía de Cádiz de Carlos Francisco Amellér (1788) fue el primer libro que se publicó en el Real Colegio de Medicina y Cirugía de Cádiz. Iba unido a la traducción de Nollet por parte de Zacagnini, pues representaba un extracto de la obra del abate francés a la que antepuso unos Elementos de Geometría, "sin cuyo estudio quedaría desconocida la mayor parte de la Física» (p. 7).

Los Eléments de Physique de La Fond, en su versión traducida al español por Tadeo Lope -ingeniero y profesor de Arte Militar y Delineación en el Real Seminario de Nobles de Madrid y autor del Curso de Matemáticas: para la enseñanza de los caballeros seminaristas del Real Seminario de Nobles de Madrid (1794-1798) - tuvieron una gran trascendencia en la difusión de la Física Experimental en España e incluso en su enseñanza, pues parece ser que se empleó en los Reales Estudios de San Isidro (García y Bertomeu, 2001, p. 355).

La Fond fue discípulo y sucesor de Nollet en la cátedra de Anatomía, Fisiología y Física experimental de París (Balpe, 1999, p. 243) y miembro de la Société Royale des Sciences de Montpellier. Interesado por los instrumentos y el funcionamiento de un laboratorio de Física experimental, creó su propio laboratorio ${ }^{21}$ en un momento en que, tras la expulsión de los jesuitas, la falta de un gran número de profesores de enseñan- za secundaria provocada por la desorganización de los collèges redundó en un aumento de las clases particulares, tanto en París como en las provincias. En su obra Description et usage d'un cabinet de physique expérimentale $^{22}$ (1975) se demostraba su interés por los instrumentos y el funcionamiento de un laboratorio de Física experimental.

\section{LA LENGUA DE LOS TEXTOS}

El vaciado terminológico de los textos objeto de análisis nos ha proporcionado un listado de términos referentes a aparatos empleados en las experimentaciones físicas, de voces referidas a objetos que formaban parte de los aparatos usados y, finalmente, de descripciones de aparatos sin denominación. Estas dos últimas categorías de términos, si bien nos permitirán establecer de un modo más completo la fisonomía característica de los textos analizados en estudios sucesivos, no forman parte del corpus de análisis de este trabajo. Asimismo, hemos recogido las ilustraciones de los aparatos cuando ha sido posible, pues cabe tener en cuenta que

el siglo XVIII fue el de los tratados técnicos ilustrados con un sinnúmero de dibujos de instrumentos científicos y de herramientas para artesanos, planes de laboratorios y fábricas o talleres, figuras anatómicas y detalles botánicos. Muy representativas de tales libros son las obras del abate Nollet (1700-1770), que se presentaban bajo la forma de pequeños volúmenes portátiles ilustrados con láminas, a veces desplegables (Clément, 1993, pp. 51-52).

Tras el estudio de los textos, nuestro corpus cuenta con 102 términos relativos a aparatos o máquinas ${ }^{23}$ (Tabla 2).

Entre los términos de la tabla 2, se puede hallar casos de variantes denominativas de dos tipos:

- Variantes denominativas sinonímicas: baroscopio/barómetro, telescopio astronómico/telescopio de Galileo, telescopio de refracción/telescopio de Newton y telescopio newtoniano o palanca/vecte.

- Variantes denominativas ortográficas: termometro, termométro, thermometro, thermómetro ${ }^{24}$.

Así mismo, es posible encontrar ejemplos en los que la variación denominativa se produce por una variación en el aparato introducida por el científico que le da nombre: barómetro simple e inclinado del Caballero Morland, barómetro simple e inclinado de M.M. Cassini y Bernouilli, hygrometro del P. Magnan, hygrometro de Sturme, hygrometro del P. Mersenne, hygrometro de los Académicos de Florencia, hygrometro de Duluc. Estos términos se refieren a aparatos concretos que ciertas personas podían utilizar en sus propios gabinetes para realizar los experimentos que 
Tabla 2. Términos de aparatos en los textos de Física experimental del siglo XVIII

\begin{tabular}{|c|c|c|c|}
\hline anteojo & anteojo de larga vista & aparato de M. Hauxbée & areómetro \\
\hline balanza hidrostática & balanza romana & barómetro & barómetro compuesto \\
\hline barómetro de Hyghens & barómetro doble de Quadrante & barómetro encogido de Ozanam & barómetro luminoso \\
\hline barómetro simple & $\begin{array}{l}\text { barómetro simple e inclinado de M.M. } \\
\text { Cassini y Bernouilli }\end{array}$ & $\begin{array}{l}\text { barómetro simple e inclinado del } \\
\text { Caballero Morland }\end{array}$ & baroscopio \\
\hline binóculo & bomba & bomba atractiva & bomba atraente \\
\hline bomba atraente é impelente & bomba atraente y compresiva & bomba atraente y elevatoria & $\begin{array}{l}\text { bomba atraente, compresiva y con } \\
\text { depósito de aire }\end{array}$ \\
\hline bomba compresiva & bomba compuesta & bomba de Céliers & bomba elevatoria \\
\hline bomba simple & caña de viento & embudo mágico & escopeta de viento \\
\hline eudiometro & fuente de compresión & fuente de mando & fuente intermitente \\
\hline horno de reverbero & hygrometro & hygrometro de Duluc & $\begin{array}{l}\text { hygrometro de los Académicos de } \\
\text { Florencia }\end{array}$ \\
\hline hygrometro de Sturme & hygrometro del P. Magnan & hygrometro del P. Mersenne & hygrometro regular \\
\hline hygroscopio & linterna catoptrico dioptrica & linterna mágica & longo-mira \\
\hline $\begin{array}{l}\text { máquina (del Padre Sebastián, } \\
\text { Carmelita Descalzo) }\end{array}$ & máquina compuesta & máquina de Archimedes & máquina de Atwood \\
\hline máquina de Boyle & máquina del Diluvio (del Abate Lebrun) & máquina hidráulica & máquina hidráulica (De Vera) \\
\hline máquina pneumática & máquina Simple & micrómetro & microscopio \\
\hline microscopio compuesto & microscopio simple & microscopio solar & monóculo \\
\hline nociometro & palanca & péndulo & péndulo compuesto \\
\hline péndulo Simple & perpendículo & pie de rey de Paris & pirómetro \\
\hline plano inclinado & polemoscopio & prisma & probatorio de la máquina neumática \\
\hline sonómetro & telescopio & telescopio astronómico & telescopio de Galileo \\
\hline telescopio de Newton & telescopio de refracción & telescopio gregoriano & telescopio holandés \\
\hline telescopio newtoniano & termometro & termométro & termométro de Delisle \\
\hline termométro de Drebbel & termométro de Fareinheit & termométro de Hauxbée & termométro de Newton \\
\hline termométro preparatorio & thermometro & thermómetro & thermómetro de Florencia \\
\hline thermometro de M. de Reamur & torno & tubo capilar & tubo de Toricelli \\
\hline vecte & ventilador (de Desaguilliers) & & \\
\hline
\end{tabular}

se describen en los textos analizados. Ante el uso de un instrumento determinado en un gabinete, los físicos podían incluir mejoras en los aparatos ya existentes para solucionar las posibles imperfecciones que pudieran presentar, o para adecuarlos a un nuevo experimento para el que no se hubieran utilizado anteriormente.

Es importante señalar la distribución de estas primeras documentaciones de voces de aparatos de Física experimental en los textos fundamentales del siglo XVIII, para después poder contrastar estos datos con la primera documentación lexicográfica. De los 102 términos extractados, 3 se documentan en Herrero y Rubira (1738), 5 en Piquer (1745), 9 en Juan y Ulloa (1748), 30 en Nollet (1757), 6 en Amellér (1788) y 49 en La Fond (1799), dato que corrobora el mayor carácter experimental de los dos textos más importantes de la época, el de Nollet y el de La Fond.

\subsection{Documentación de los términos en el CORDE}

El motivo de la búsqueda de estos términos en el CORDE se halla en la finalidad misma del corpus, pues, como expone la RAE en la sección de Ayuda del Banco de datos del español, pretende servir tanto a un investigador interesado en la existencia de una palabra o expresión o que quiera llevar a cabo un estudio gramatical, como a los lexicógrafos que con sus materiales elaboren el Diccionario histórico.

En la consulta que hemos realizado para este estudio, se ha podido constatar que, por lo que respecta al ámbito de la Física experimental, y, en concreto, en lo que atañe a los aparatos y las máquinas que los científicos utilizaban en sus experimentos, el $83,4 \%$ de los 102 términos documentados en los textos estudiados no se encuentra en las obras que recoge el CORDE. Este dato demuestra que este corpus presenta carencias en cuanto a su selección para estudiar la historia del léxico científico y técnico español, a pesar de que se trata de un corpus muy amplio de textos, en la línea de lo que han demostrado otros estudios similares al nuestro ${ }^{25}$.

Si nos centramos en el $16,6 \%$ de términos que sí se recoge en el corpus académico ${ }^{26}$, resulta muy interesante saber que la única documentación que se realiza en el CORDE en un texto anterior al siglo XVIII es la del término palanca. Aparece en la Traducción de la Mechánica de Aristóteles, de Diego Hurtado de Mendoza (1545) en 38 ocasiones, lo que nos parece lógico puesto que la palanca es una de las piezas fundamentales de la mecánica desde la Antigüedad. 
En cuanto a los términos registrados en el CORDE por primera vez en textos del siglo XVIII, estos representan el $4,9 \%$ del corpus analizado y son los siguientes: horno de reverbero, hygrometro, microscopio, termométro de Fareinheit y plano inclinado. Todos se documentan en los Anales del Real Laboratorio de Química de Segovia de Proust (1791) y en el Theatro crítico universal de Feijoo (1728), excepto termométro de Fareinheit que se cita en Geografía física y esférica de las provincias del Paraguay de Félix de Azara (1790).

En textos del siglo XIX se documentan por primera vez 7 términos $(6,86 \%)$ :

- anteojo, areómetro, barómetro, bomba, prisma y máquina pneumática en el Manual de física popular de Vicuña (1881).

- linterna mágica, prisma y máquina pneumática en el Manual de electricidad popular de Casas Barbosa (1881).

$\mathrm{Y}$ en textos del siglo $\mathrm{XX}$, se pueden hallar 4 términos (3,92\%). La mayoría de ellos (péndulo, péndulo simple, péndulo compuesto) se citan de la obra Elementos de física general de Sanjurjo (1910).

Los datos expuestos se ilustran en la tabla 3.

Además, la mayoría de términos se recopila a partir de un número de textos limitado, lo que nos permite afirmar que las fuentes básicas en las que se han documentado los términos en el CORDE han sido las siguientes:

- para el siglo XVIII, los textos de Proust y del Padre Feijoo;

- para el siglo XIX, los textos de Vicuña y Barbosa;

- y para el siglo XX, el texto de Sanjurjo.
La conclusión a la que nos conducen estos resultados es que el CORDE no puede sustituir al análisis de los textos científicos específicos del período histórico objeto de estudio con el fin de determinar la primera documentación de los términos para incluirlos, posteriormente, en el Nuevo Diccionario Histórico de la Lengua Española de la Real Academia Española. Por tanto, nuestra investigación permite ampliar la nómina de autores y de obras fundamentales que aparece en el CORDE para el estudio del léxico de la ciencia y la técnica del siglo XVIII en lo que respecta al ámbito de la Física experimental, hecho que redundará, sin duda, en un mejor conocimiento tanto de los textos científicos más relevantes para la Historia de la ciencia como a la hora de establecer la historia de los términos objeto de análisis.

\subsection{Documentación lexicográfica de los términos}

El paso siguiente a la documentación de las voces en los textos fundamentales del siglo XVIII y a su contraste con los datos recogidos en el CORDE lo representa el análisis de la incorporación de los términos del corpus en los diccionarios generales españoles, para documentar su primera entrada en un diccionario de lengua española y sentar, así, las bases para la realización del DHEMAFE, integrado en el DHEMCYT. Para ello, hemos realizado un rastreo de los 102 términos en los diccionarios incluidos en el NTLLE.

Los resultados de este análisis indican que 49 de los 102 términos extractados $(48,04 \%)$ no se recogen en ninguno de los diccionarios consultados ${ }^{27}$. Entre ellos, se encuentran los que se caracterizan por su especialización, como linterna catoptrico dioptrica (Amellér, 1788), probatorio de la máquina neumática (La Fond, 1799) o tubo de Toricelli (Juan y Ulloa, 1748). Hay que tener en cuenta que, en muchos casos, estos términos

Tabla 3. Los términos documentados en el CORDE

\begin{tabular}{cccccc}
\hline & $\begin{array}{c}\text { anterior al } \\
\text { s. XVIII }\end{array}$ & s. XVIII & s. XIX & s. XX & TOTAL \\
\hline No se documentan en el CORDE & 0 & 0 & 0 & 0 & $85(83,4 \%)$ \\
\hline Se documentan en el CORDE & 1 & 5 & 7 & 4 & $17(16,6 \%)$ \\
\hline
\end{tabular}


solo aparecen en estos textos específicos no considerados por los lexicógrafos, precisamente por su alto grado de especialización. Tampoco se documentan lexicográficamente términos que incluyen el nombre del autor, como en thermometro de $M$. de Reamur (Juan y Ulloa, 1748), termométro de Drebbel (La Fond, 1799), termométro de Delisle (La Fond, 1799), termométro de Hauxbée (La Fond, 1799), termométro de Newton (La Fond, 1799). A este respecto, el término termometro se documenta ya en Sobrino (1705), con la variante sin tilde y más tarde en Terreros (1788), también sin tilde, pero los distintos tipos de termómetros que se manejan para los experimentos en los gabinetes de Física, creados por los distintos autores que se citan en las denominaciones, no aparecen en la nomenclatura de los diccionarios estudiados, ni tampoco como subentradas bajo el término base que sí se recoge en la mayoría de ellos. En cambio, se produce una mención de los aparatos a los que se hace referencia con estas denominaciones en el desarrollo explicativo de los diccionarios enciclopédicos, que normalmente son los que incorporan este tipo de explicaciones. Así, puede verse, por ejemplo, en la documentación de la voz termometro en el Diccionario Castellano con las voces de ciencias y artes de Terreros ${ }^{28}$ :
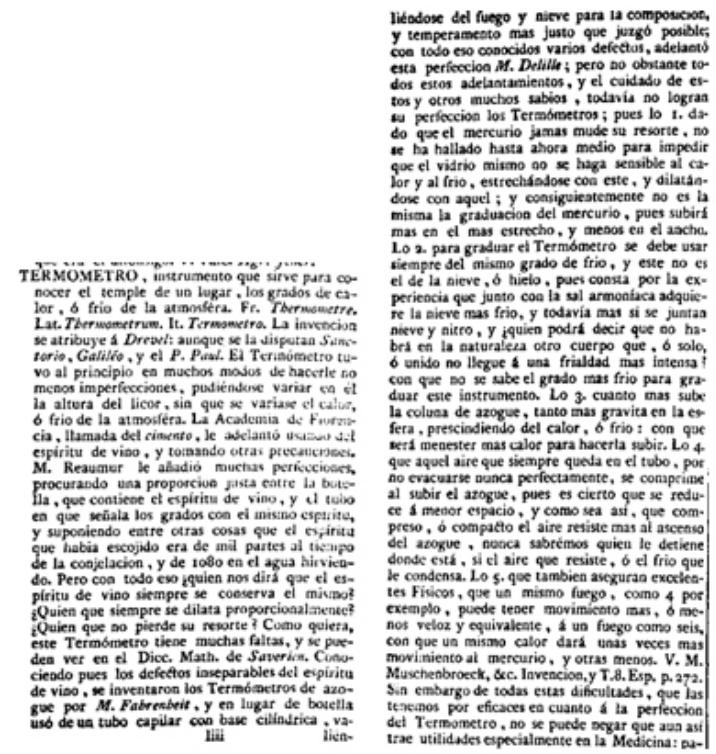

ra coya ineligencia potare aqui brevemente

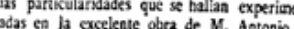

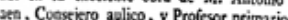
Medicina prética en la Uriversidad de Vix cyyo tik. es: Ratio mededendi in Nasocomio prac

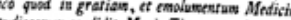

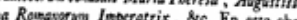

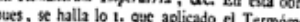

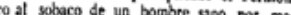
curto de bota, sube at grado de os. á of che Calor, y aplicado ua cuartio de hora sibe del go or thas unarhora no pasa del 102 , sin

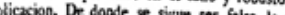

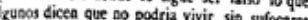

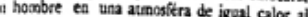

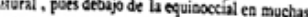

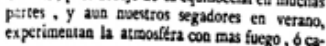
99 gt. y por media hora del 100 , al 101 ,

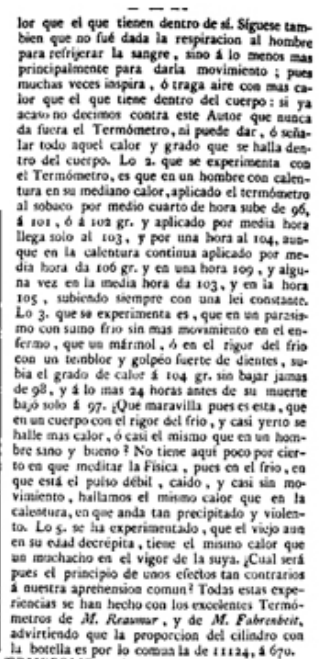

(Nollet, 1757); y 2 en el siglo XX, fuente (DRAE-1984) $)^{30}$ y telescopio de refracción (DRAEM-1985) (Nollet, 1757).

Por último, de los términos que se documentan lexicográficamente por primera vez en diccionarios no académicos, destacan, por una parte, 8 que se recogen por primera vez en Terreros -areómetro (Nollet, 1757), baroscopio (Nollet, 1757), máquina de Arquímedes (Herrero y Rubira, 1738), micrómetro (Juan y Ulloa, 1748), pie de Rey (Juan y Ulloa, 1748), pirómetro (Nollet, 1757), polemoscopio (Nollet, 1757), vecte (Amellér, 1788) y ventilador (La Fond, 1799)-. Por otra parte, sobresalen 11 voces que se registran por vez primera en diccionarios no académicos de mitad del siglo XIX: en el Diccionario Nacional (184647) de R. J. Domínguez - balanza hidrostática (Nollet, 1757), bomba atraente e impelente (La Fond, 1799), eudiometro (La Fond, 1799), fuente de compresión (La Fond, 1799), fuente intermitente (La Fond, 1799), máquina compuesta (Amellér, 1788), máquina simple 
(Amellér, 1788), sonómetro (La Fond, 1799) y tubo capilar (Amellér, 1788)- y en el Diccionario enciclopédico de la lengua española (1853-55) de Gaspar y Roig -bomba atraente e impelente (La Fond, 1799), péndulo compuesto (La Fond, 1799) y péndulo simple (La Fond, 1799)-

Algunos de estos términos (balanza hidrostática, baroscopio, máquina de Arquímedes, máquina compuesta, máquina simple, péndulo compuesto, péndulo simple ${ }^{31}$, polemoscopio y vecte) no se incorporaron al $D R A E$ en ninguna de sus ediciones. Otros, lo hicieron a lo largo del siglo XIX: areómetro, micrómetro en su edición de 1817; pirómetro en 1822; ventilador en 1843; bomba atraente e impelente y eudiómetro en 1884; otros a lo largo del siglo XX: sonómetro y fuente en 1984; tubo capilar en 1985 (Manual) y, por último, pie de rey se ha incorporado en la edición de 2001, la última, por ahora, del diccionario académico.

De todas estas documentaciones en los diccionarios académicos, es llamativo el hecho de que términos como baroscopio (Nollet, 1757) no lleguen a entrar en el DRAE y que otros como eudiómetro (La Fond, 1799) lo hagan a principios del XIX. Incluso cabe destacar que un término documentado ya en un texto del siglo XVIII como pie de Rey (Juan y Ulloa, 1748) se acabe incorporando en el diccionario en su primera edición del siglo XXI, tres siglos más tarde.

Como complemento de los datos lexicográficos expuestos en los diccionarios generales de lengua que se recogen en el NTLLE, hemos consultado las voces extractadas en diccionarios científicos y técnicos de comienzos del siglo XIX. Cabe mencionar que no se halla ningún diccionario de Física experimental, ni en el siglo XVIII ni en el XIX, motivo por el cual hemos recurrido a observar el Diccionario universal de Física de Brisson (1796-1802), en su traducción al español, y dos repertorios de ciencias experimentales que, como los de Medicina y Farmacia del siglo XIX, podían considerarse interdisciplinares con la Física experimental: el Nuevo Diccionario español e inglés que contiene la etimología, de la propia, y metaphorica significación de las palabras, términos de artes y sciencias, de Pedro Pineda (Londres, 1740) y el Vocabulario médicoquirúrgico, o Diccionario de Medicina y Cirugia, que comprende la etimologia y definicion de todos los terminos usados en estas dos ciencias por los autores antiguos y modernos, de Hurtado de Mendoza (Madrid, 1840). Estas dos obras, junto con la de Brisson, abarcan de mediados del siglo XVIII a mediados del siglo XIX, época de plena difusión de los términos expuestos en este trabajo.

Tras el análisis de las voces objeto de estudio en estas obras, observamos, en primer lugar, que se adelanta la datación lexicográfica de algunas de las voces en relación con los diccionarios de lengua recogidos en el NTLLE y, en segundo lugar, que se documentan voces que no documentamos en textos lexicográficos generales. Por lo que respecta al primer aspecto, se adelanta la datación lexicográfica de 11 voces (eudiómetro, fuente intermitente, hygroscopio, máquina de Boyle, máquina pneumática, microscopio solar, péndulo, péndulo compuesto, péndulo simple, polemoscopio y sonómetro), todas ellas documentadas por primera vez en el Diccionario universal de Física de Brisson, como es esperable al tratarse de un diccionario de Física. En cuanto a la documentación lexicográfica de voces que no se documentaban en repertorios generales, se han podido documentar, también en el Diccionario universal de Física de Brisson, 19 voces (barómetro simple, barómetro de quadrante, barómetro luminoso, bomba atractiva, bomba compresiva, hygrometro de Duluc, nociómetro, telescopio astronómico, telescopio de Galileo, telescopio gregoriano, telescopio newtoniano, termométro de Fareinheit, termométro de Reamur, termométro de Florencia, termométro de Drebbel, termométro de Delisle, termométro de Newton, tubo capilar y tubo de Toricelli).

Los datos definitivos de los diccionarios estudiados, nos revelan que 32 de los 102 extractados (31,37\%) no aparecen en ninguno de los diccionarios analizados, sean generales, sean especializados, lo cual demuestra que es importante tener en cuenta la información recogida en los diferentes diccionarios para el estudio y conocimiento de las voces, al recogerse 70 términos, pero asimismo refuerza la necesidad de trabajar desde los textos fundamentales de la época para aportar términos usados y difundidos pero no recopilados en los diccionarios.

\section{CONCLUSIONES}

El proceso de documentación de las voces es complejo, pues hay que tomar en consideración muchas variables: palabra, texto, autor, período, etc. Para poder determinar la repercusión de las palabras de nuestro corpus, es necesario conocer los textos fundamentales, en nuestro caso, por medio de su empleo en instituciones más o menos prestigiosas o a partir de la importancia de sus autores. Más concretamente, los resultados del análisis de los datos demuestran que, por lo que respecta a la documentación de voces referentes a aparatos de Física experimental del siglo XVIII, los textos analizados son fundamentales porque ejercieron una influencia decisiva en el desarrollo de la Física experimental en España y permitieron divulgar neologismos, en ese período, referentes a aparatos. Las traducciones de Nollet y La Fond por parte de Zacagnini y Lope, respectivamente, sentaron las bases para la introducción y posterior divulgación de la Física experimental en España. Por todo ello, el estudio del léxico de la ciencia y la técnica del siglo XVIII debe tener en consideración los textos estudiados; más 
concretamente, para el ámbito de la Física experimental, sin estos textos cualquier intento de realizar una historia de la lengua o una obra lexicográfica de carácter histórico resultaría, cuando menos, incompleto.

Conocer la Historia de la Ciencia se hace imprescindible para poder establecer una verdadera «historia de las palabras» pertenecientes a la Física Experimental si se pretende acometer el estudio de los textos científicos de la época con el rigor que se requiere para un estudio de este tipo.

\section{NOTAS}

1 Este estudio se inserta en el marco del proyecto de investigación Diccionario histórico del español moderno de la ciencia y de la técnica (fase avanzada), desarrollado por el grupo NEOLCYT (http://seneca.uab.es/neolcyt), Grupo Consolidado de la Generalitat de Catalunya (2009SGR-937) y financiado por el Ministerio de Educación y Ciencia (FFI2010-15240). Este grupo participa en la Red Temática «Lengua y ciencia» (FFI2009-05433-E).

2 Para la importancia de Musschenbroek en la Física experimental del siglo XVIII, véase Guijarro Mora (2001b).

3 A este respecto, véanse, entre otros, los estudios de Gutiérrez Rodilla (1998), Gutiérrez Cuadrado (2001), Gutiérrez Cuadrado (2004), Gómez de Enterría (2003), Messner (2004), Battaner y Borrás (2004) o Rodríguez y Garriga (2006). No obstante, existen traducciones de otros idiomas, como del italiano, tal y como se expone en Gutiérrez Cuadrado (1998).

4 Para más detalles, véase Gutiérrez Cuadrado (2004)

5 Para mayor información sobre la militarización de la ciencia, véase Lafuente y Peset (1982 y 1985).

6 Con la expulsión de los jesuitas (1767), pudo terminarse con el escolasticismo reinante (Maldonado y García, 2002).

7 En España, la Real Academia de Ciencias Exactas, Físicas y Naturales no se creó hasta 1847 . Para más detalles, véase Clément (1993, pp. 34-35)

8 La fecha entre paréntesis indica la traducción de Antonio Colón Zacagnini al español del original Leçons de physique expérimentale (1743-48).

9 Este método se llevaba a cabo «mediante la observación y el experimento de esos nuevos campos de la naturaleza que suponían los fenómenos del calor, de la electricidad y del magnetismo» (Sellés, 2001, p. 163).

10 Para una aproximación a la selección de textos de Física experimental de los siglos XVIII y XIX, véase Nomdedeu (2012b).

11 Tenemos noticias, por ejemplo, de que los más de 80 instrumentos que formaron parte del gabinete de Física experimental del Colegio de Minería en México se tomaron de los Elementos de Física Teórica y Experimental de La Fond (1799) (Ramos Lara, 1999).
El hecho de que los textos de nuestro corpus de análisis recojan un porcentaje de términos no documentados muy elevado tanto en los diccionarios $(31,37 \%)$ como en el CORDE $(83,4 \%)$ refuerza la necesidad de continuar en esta línea de investigación que desencadenará en la elaboración del DHEMAFE como parte del DHEMCYT. Solo así podremos trazar una historia del léxico científico y técnico español adecuada a la realidad del uso de las voces.

12 Discrepamos de Miralles y Miralles (2007) al considerar el libro de Piquer como el primer manual de Física escrito en español. Siete años antes de la aparición de este tratado, Antonio M. Herrero y Rubira publica Physica moderna, experimental, sistematica.

13 De acuerdo con los datos recogidos en la Biblioteca Nacional de París. La última publicada en 1786.

14 Para más detalles sobre la obra de Nollet en español, véase Nomdedeu (2012a)

15 La Royal Society of London for Improving Natural Knowledge ('Sociedad Real para el Avance de la Ciencia Natural') (http:// royalsociety.org/) es la sociedad científica más antigua del Reino Unido y una de las más antiguas de Europa.

16 La Academia de las Ciencias de Francia (http://www.academiesciences.fr/), creada en 1666, durante el reinado de Luis XIV bajo el patrocinio de su primer ministro Jean-Baptiste Colbert, contó inicialmente con científicos como René Descartes, Blaise Pascal y Pierre de Fermat. Se trata de una de las cinco academias francesas que constituyen el actual Instituto de Francia. En 1699, recibe el título de «Real». Estudiado por Moreno Villanueva (1996).

17 En 1739, el rey de Cerdeña lo llamó a Turín para instruir al duque de Saboya y para proporcionar los instrumentos necesarios para la nueva cátedra de Física de la universidad que no se crearía hasta 1820 para Amadeo Avogrado.

18 Como traductor, aparte de la obra objeto de estudio, tradujo todas las demás obras de Física de Nollet, que quedaron inéditas en su seminario, hizo traducciones en verso de las tragedias que se representaban en el seminario, como las Sedecias del padre Granelli. Tradujo también La muerte de César de Voltaire (De Cambiaso y Verdes, 1829, pp. 244-245).

19 El Seminario de Nobles de Madrid, creado por Felipe V en 1725 , «destinado a la educación de la nobleza, desde mediados de la centuria el Seminario inició una lenta apertura hacia las «capas burguesas» de la sociedad, proceso que corrió paralelo a su progresiva «militarización». No obstante, la mayor parte de sus alumnos procedían de familias de la nobleza media y baja, de la administración del Estado, del ejército y de las oligarquías locales» (Andújar, 2004, p. 201). 
20 Pasado el tiempo, y habiendo recibido la influencia de Nollet a través de Zacagnini, el Real Colegio de Cirugía de Cádiz se dotó de textos de Física experimental, siendo el primero los Elementos de geometría y física experimental de Carlos Francisco Ameller en 1788, usado como manual y obra de referencia en numerosas universidades del país. Le siguieron las Lecciones elementales de Física experimental con aplicación a la medicina y a las artes de José María López y López de 1835 y las Lecciones de física médica de Manuel Losela de 1845 de unas lecciones dictadas por José Gardoqui.

21 En http://s.boudreux.free.fr/cabinet_Sigaud puede encontrarse información sobre este primer laboratorio.

22 Sigaud de La Fond fue un autor prolífico en cuanto a escritos relacionados con la Física experimental: Leçons de physique experiméntale (1767), la traducción de los tres volúmenes de los Cours de physique experimental et mathématique de Musschembroek, Traité de l'Electricité (1771), Dictionnaire de physique (1781), Précis historique et expériences des phénomènes électriques depuis l'origine de cett découverte jusqu'à ce jour (1785), Examen de quelques principes erronés en électricité (1796) y De l'électricité médicale (1803).

23 Puesto que se trata de denominaciones de objetos que a lo largo de su existencia han sufrido modificaciones, es posible hallar casos en los que la variación denominativa del término viene motivada por una modificación en el aparato introducida por el científico que le da nombre, como en el caso de Hygrometro: del P. Magnan, de Sturme, del P. Mersenne, de los Académicos de Florencia y de Duluc. También se han podido documentar variantes denominativas sinonímicas, como en baroscopio/barómetro y variantes denominativas ortográficas, como en termometro, termométro, thermometro, thermómetro.

24 Cabe señalar que en los textos de Física experimental estudiados no se documenta la voz termómetro tal y como se escribe actualmente. Habrá que esperar a la documentación del térmi-

\section{BIBLIOGRAFÍA}

Andújar Castillo, Francisco (2004), "El Seminario de Nobles de Madrid en el siglo XVIII. Un estudio social", Cuadernos de Historia Moderna. Anejos, III, pp. 201-225.

Balpe, Claudette (1999), "Constitution d'un enseignement experimental: La physique et chimie dans les écoles centrales", Revue d'Histoire des sciences, 52 (2), pp. 241-284.

Battaner Arias, Paz y Borrás, Laura (2004), "Traducciones y adaptaciones de diccionarios y obras de historia natural en el siglo XIX". En: Alsina, Victòria; Brumme, Jenny; Garriga, Cecilio y Sinner, Carsten (eds.), Traducción y estandarización. La incidencia de la traducción de los lenguajes especializados, Madrid-Frankfurt am Main, Iberoamericana-Vervuert, pp. 169-191.

Clément, Jean-Pierre (1993), Las instituciones científicas y la difusión de la ciencia durante la ilustración, Madrid, Akal. no en textos de esta disciplina del siglo XIX, que abordaremos en estudios posteriores.

25 Véase Rodríguez y Garriga (2006)

26 Este porcentaje corresponde a los 17 términos siguientes: anteojo, areómetro, barómetro, bomba, bomba atraente e impelente, horno de reverbero, hygrometro, linterna mágica, máquina pneumática, microscopio, palanca, péndulo, péndulo compuesto, péndulo simple, plano inclinado, prisma y termométro de Farenheit.

27 En este apartado, indicamos al lado de cada término y entre paréntesis su primera documentación en un texto de Física experimental.

28 En el diccionario de Gaspar y Roig también se menciona la máquina de Atwood y la máquina de Boyle en el desarrollo enciclopédico correspondiente a la biografía de los dos autores, Atwood y Boyle, en la que se dice que construyeron sendas máquinas de las que se explica su uso.

29 Anteojo (Nollet, 1757), binóculo (Nollet, 1757), escopeta de viento (La Fond, 1799), linterna mágica (Nollet, 1757), microscopio simple (Nollet, 1757), microscopio compuesto (Nollet, 1757), palanca (Piquer, 1745), perpendículo (Juan y Ulloa, 1748), plano inclinado (La Fond, 1799), prisma (Nollet, 1757) y telescopio (Nollet, 1757).

30 Aparece una acepción marcada como Fís. pero no se especifica ninguna de los tres tipos de fuentes que hemos documentado en los textos. En el DRAE de 1992 desaparece esta acepción marcada.

31 EI DRAE-1817 incorpora la acepción (marcada) correspondiente a la Estática, que se correspondería con el sentido que nosotros hemos documentado, pero en ningún caso se hace distinción entre estos dos tipos de péndulos.
De Cambiaso y Verdes, N.M. (1829), Memorias para la Biografía y para la Bibliografía de la Isla de Cádiz. Madrid, Imprenta de D. Leon Amarita, tomo primero, pp. 244-245.

García Belmar, Antonio y Bertomeu Sánchez, José Ramón (2001), "Pedro Gutiérrez Bueno (1745-1822), los libros de texto y los nuevos públicos de la química en el último tercio del siglo XVIII", DYNAMIS. Acta Hisp. Med. Sci. Hist. Illus, 21, pp. 351-374.

Gómez de Enterría, Josefa (2003), “Notas sobre la traducción científica y técnica en el siglo XVIII", Quaderns de Filología. Estudis Lingüístics, Historia de la Traducción, VIII, pp. 35-67.

González de Posada, Francisco (2008), “Jorge Juan: innovador de la Educación Superior en la España ilustrada”, Revista Complutense de Educación, 19 (1), pp. 115-135. 
Guijarro Mora, Víctor (2001a), "La enseñanza de la física experimental en la Europa del siglo XVIII", ÉNDOXA: Series Filosóficas, 14, pp. 111-136.

Guijarro Mora, Víctor (2001b), "Petrus Van Musschenbroek y la física experimental del siglo XVIII", Asclepio, LIII (2), pp. 191-212.

Gutiérrez Cuadrado, Juan (1998), "F. Carbonell y Bravo y su texto Curso analítico de química escrito en italiano por F. Mojón". En: García Turza, Claudio; González Bachiller, Fabián y Mangado, José Javier (eds.), Actas del IV Congreso Internacional de Historia de la Lengua Española, Logroño, AHLE-Gobierno de La RiojaUniversidad de La Rioja, pp. 219-230.

Gutiérrez Cuadrado, Juan (2001), "Lengua y ciencia en el siglo XIX español: el ejemplo de la química”. En: Bargalló Escrivà, María; Forgas Berdet, Esther; Garriga Escribano, Cecilio; Rubio, Ana y Schnitzer, Johannes (eds.), Las lenguas de especialidad y su didáctica, Tarragona, Universitat Rovira i Virgili, pp. 181-196.

Gutiérrez Cuadrado, Juan (2004), "Las traducciones francesas, mediadoras entre España y Europa en la lengua técnica del siglo XIX". En: Alsina, Victòria; Brumme, Jenny; Garriga, Cecilio y Sinner, Carsten (eds.), Traducción y estandarización. La incidencia de la traducción de los lenguajes especializados, MadridFrankfurt am Main, Iberoamericana-Vervuert, pp. 35-60.

Gutiérrez Rodilla, Bertha (1998), La ciencia empieza en la palabra. Análisis e historia del lenguaje científico, Barcelona, Ediciones Península.

Lafuente, Antonio y Peset, José Luis (1982), "Las Academias Militares y la inversión en ciencia en la España ilustrada (1750-1760)", Acta Hispanica ad Medicinae Scientiarumque Historiam Illustrandam, 2, pp. 193-209.

Lafuente, Antonio y Peset, José Luis (1985), “Militarización de las actividades científicas en la España ilustrada (1726-1754)". En: Peset, José Luis (ed.), La ciencia moderna y el Nuevo Mundo, Madrid, CSIC, pp. 127-147.

Maldonado Polo, José Luis y García González, Armando (2002), La España de la técnica y la ciencia. Madrid, Acento Editorial.

Messner, Dieter (2004), “La traducción de textos franceses de especialidad a las lenguas iberorrománicas en el siglo XVIII". En: Alsina, Victòria; Brumme, Jenny; Garriga, Cecilio y Sinner, Carsten (eds.), Traducción y estandarización. La incidencia de la traducción de los lenguajes especializados, Madrid-Frankfurt am Main, Iberoamericana-Vervuert, pp. 19-33.

Miralles Conesa, Luis y Miralles Hernández, M.a José (2007), “La enseñanza de la física en el siglo XVIII: La física moderna, racional y experimental (1745) de Andrés Piquer Arrufat" (primer libro de física escrito en español), Didáctica de las ciencias experimentales y sociales, 21, pp. 169-196, [en línea], disponible en: http:// dialnet.unirioja.es/servlet/oaiart?codigo $=2476027$, [consultado el 26/08/2013]
Moreno Villanueva, José Antonio (1996), "Jean-Antoine Nollet y la difusión del estudio de la electricidad: un nuevo léxico para una nueva ciencia", DOCUMENTS pour I'histoire du français langue étrangère ou seconde, 18, pp. 405-417, [en línea], disponible en: http://dfe.uab.cat/neolcyt/images/stories/estudios/electricidad/mor1996.pdf, [consultado el 26/08/2013].

Nomdedeu Rull, Antoni (2012a), “La creación del léxico de los aparatos de Física experimental en español: Jean Antoine Nollet y Antonio Nicolás Zacagnini", Revista de Investigación Lingüística, 15, pp. 227-253.

Nomdedeu Rull, Antoni (2012b), “Notas sobre el vocabulario de la física experimental en español en los textos fundamentales de los siglos XVIII y XIX". En: Montero Castelle, Emilio (ed.), Actas del VIII Congreso Internacional de Historia de la Lengua Española, Santiago de Compostela, Meubook, 2012, tomo II, pp. 1513-1528.

Peset, José Luis y Lafuente, Antonio (1981), “Ciencia e historia de la ciencia en la España ilustrada", Boletín de la Real Academia de la Historia, 178, pp. 267-300.

Ramos Lara, M.a de la Paz (1999), "La enseñanza de la física en México en el siglo XVIII. El proceso de institucionalización", Revista Mexicana de Física, 45 (2), pp. 193-203.

[CORDE] Real Academia Española: Banco de datos (CORDE), Corpus diacrónico del español, [en línea], disponible en: http://www. rae.es [consultado el 26/08/2013].

[NTLLE] Real Academia Española (2001), Nuevo Tesoro lexicográfico de la lengua española, DVD.

Rodríguez, Francesc y Garriga Escribano, Cecilio (2006), "La lengua de la ciencia y la técnica moderna en el CORDE: los Anales de Química de Proust". En: Bernal, Elisenda y DeCesaris, Janet (eds.), Palabra por palabra: estudios ofrecidos a Paz Battaner, Barcelona, IULA-UPF, pp. 219-232.

Rodríguez Ballesteros, Juan J. (2004), "La introducción de la Física en los estudios médico-quirúrgicos y en la Armada gaditana", LLULL, 27, pp. 475-493.

Sánchez Ron, José María (1988), Ciencia y sociedad en España, de la Ilustración a la Guerra Civil, Madrid, El Arquero.

Sellés, Manuel A. (2001), "El vapor en el laboratorio: una memoria sobre la ebullición del abate Nollet", Asclepio, LIII (2), pp. 165189.

Ten Ros, Antonio Enrique (1983), "La física experimental en la universidad española de fines del siglo XVIII y principios del XIX. La Universidad de Valencia y su aula de Mecánica y Física experimental", Llull, 6, pp. 165-189.

Vernet Gines, Juan (1975), Historia de la ciencia española, Madrid, Artes Gráficas Soler. 